\title{
Training of Learning Strategies for Agricultural University Non-English Majors
}

\author{
Bo Du ${ }^{\mathrm{a}}$, Jing Zhang ${ }^{\mathrm{b}}$, Yan Zhang ${ }^{\mathrm{c}}$ \\ Foreign Languages College of Agricultural University of Hebei, Baoding 071001, China \\ aDuBo80@126.com, b1301683942@qq.com, cLdmmail@163.com
}

\begin{abstract}
In linguistics, foreign language learning strategy has gradually become a focus of research. Based on an in-depth survey of 415 non-English major students in the Agricultural University of Hebei, a study has shown because of some objective reasons, such as students' source and specialty nature, the public English teaching in agricultural and forestry universities undergraduates is not satisfactory, whose main performance is that students' English autonomous learning consciousness is relatively weak, students lack of learning strategy awareness, and use frequency of strategy is low. To solve these problems, some suggestions are put forward to improve the teaching effectiveness of teachers and students.
\end{abstract}

Keywords: Agricultural and forestry universities; Learning strategies; Non-English majors; Metacognitive awareness.

\section{农林院校非英语专业英语学习策略的调查研究}

\author{
杜渤”, 张静, 张燕 \\ 河北农业大学 外国语学院, 河北 保定 071001, 中国
}

摘 要: 外语学习策略研究逐渐成为语言学界的研究重点, 本文通过问卷调查、访谈等形式对 河北农业大学415名非英语专业本科生的学习策略情况进行了调查研究。结果表明: 由于诸如 学生来源、专业性质等客观原因, 农林院校本科公共英语教学不尽人意, 主要表现为学生英 语自主学习意识相对薄弱, 缺乏学习策略意识, 策略使用频率较低。针对这些问题提出了学 习策略培训的几点建议, 使其有针对性地运用认知策略, 以提高教师的教学效果和学生的学 习效率。

关键词: 农林院校; 学习策略; 非英语专业; 元认知意识

\section{1. 引言}

外语学习策略 (Learning Strategy) 是教育心理学关于学习理论的重要内容。它是指学习者在 学习过程中采用的总的对策、措施和方法, 即以达到掌握外语为目的的学习思维和活动程序。 学习策略的研究对外语教学有着极其重要的意义, 我们日益意识到: 不管我们的教学方法在 理论上如何完善却难以产生理想的效果, 学习者在年龄、性别、兴趣、学能、动机、学习策 略、个性、认知方式等方面的个体差异决定了我们不可能指望依靠教师用同一方式将学生塑 造成一个模式。语言学习的成功主要在于学习者本人充分利用各种学习机会的能力, 即怎样 使用学习策略, 于是语言学界逐渐将研究重心转移到学习者身上一一由研究教师如何教转移 到探讨学生如何学。我国语言界也将学习者的个人差异 (其中包括学习策略) 和多媒体教学列 为应用语言学 (语言教学研究) 近十年的两个研究重点。

开展英语学习策略的研究, 有助于在教学中提升学生的学习策略, 培养学生的自主学习能力; 有助于学生挖掘学习潜力, 提高学习效率。对于众多的非英语专业大学生来说, 提高英语学 习策略水平尤为重要。 


\section{2. 农林院校学生英语学习现状}

河北农业大学是河北省规模最大的一所农林本科院校, 农村生源在校生比例约为 $61.7 \%$ (据 2014 年统计资料）。受专业性质的影响, 农林院校生源多来自农村, 其中很大一部分来自经 济发展相对落后的边远地区, 由于受到诸多客观条件的限制, 农村学生较之城市学生在英语 基础、学习习惯、学习方法等方面都存在很大差异。认清这种差异, 认清农村学生的英语学 习特点, 是加强农林院校公共英语教学改革针对性的关键, 也是因材施教的基本前提。因此, 我们选取河北农业大学修读英语课程的本科生为主要研究对象, 对其学生的英语学习特点进 行了调查分析。

就河北农业大学的学生而言, 英语学习状况及水平不容乐观。根据小组抽取河北农业大学 2014 级分别来自计算机、农电、生物、食品、医学、财会专业共 415 名非英语专业学生就英语学 习方面问题, 尤其是英语学习策略进行问卷调查, 共收回有效问卷为 394 份。在抽样调查的 基础上, 分析学生在学习策略上主要存在以下问题。

2.1. 学习策略贫乏

在教学过程和访谈过程中, 学生最常提到 “不知道如何学习, 如何调整学习方法” 等问题。 说明虽然他们具备了基本的学习策略。如利用工具书、背诵、通过上下文记忆等, 但是对其 它层面的学习策略了解甚少, 形象、声像、记忆关键词、发现语言规律并运用等几项认知策 略在学生们学习策略的自我评估中平均值最低。调查显示: 大部分学生课后的主要学习活动 是背单词、作练习、对答案, 只有 $12 \%$ 的人能做到经常听课文磁带, “除了教师布置的任务 外什么也不学” 的同学占 $64 \%$ 。虽然很多同学都意识到了阅读和练习造句对增加词汇量有很 大的帮助, 但是, 大多数同学还是看单词表背单词, 从来没想过把单词放在语境中来记, 每 次复习只是把单词表默读几遍, 这种认知策略的单一是造成英语学习费时多、收效低的主要 原因。

\section{2. 策略应用较少}

从统计数据看, 接近一半的学生在制定学习计划上选择通常符合, 这说明学生在学习过程中 有制定计划的习惯, 但程度并不高; 有超过三分之一的学生在学习过程中没有制定计划的习 惯; 有超过五分之一的学生在学习过程中不会利用图画等非语言信息帮助理解; 有四分之三 的学生在学习英语的过程中会去发现并利用规律。大多数学生认为阅读英语读物有助于英语 学习, 同时认为学习英语首先要明确学习目标; 有一半的学生在反思学习中的进步与不足上 选择基本同意, 说明学生有反思的意识, 但程度不高; 有 $97 \%$ 的学生觉得探索适合自己的学 习方法有必要, 这说明学生对学习方法的需求非常高, 应引起老师的关注; 同时大多数学生 认为借助工具书和电子词典有助于英语学习; 大多数学生愿意利用图书馆和网络等资源去帮 助学习英语, 但 $41.9 \%$ 选择基本同意也说明利用的程度不高。

\section{3. 对教学的启示}

农林院校本科公共英语教学之所以不太尽人意, 有诸如学生来源、专业性质等客观原因, 农 村生源的英语基础和城市生源的英语基础有显著差异, 这是我们英语教改不得不面对的现实。 考虑这一实际情况, 在培养学生学习策略方面, 作为教师应经常有意识地去发现适用于农林 院校学生的学习策略。综合起来, 主要有以下几个方面:

3.1 加强策略理论的学习

学习策略对自主学习有显著影响, 学习策略能力的形成使最终的真正意义上的自主学习成为 可能, 使用学习策略的意识越强, 自主学习的过程就越完整, 效果就越好。在英语教学中加 强学生学习策略的指导和训练, 可以有效培养学生的自主学习和终身学习的能力。因此, 在 进行学习策略训练之前, 首先要让学生认识到学习策略以及训练的意义和重要性, 增强策略 意识, 明白他们学习成绩不理想的原因并不完全是自己努力不够, 而是缺乏有效的学习策略; 学习策略训练的最终目的不是单纯地学习方法指导, 而是使他们了解自己当前的学习方式, 
知道在什么情况下使用什么策略以及如何使用策略, 帮助学生探索适合自己个性化的学习系 统。

\section{2. 重视元认知策略的培训}

元认知策略与英语学习密切相关, 而学生对于元认知策略中的自我监督、自我评估使用频率 偏低。产生这问题的主要原因在于以教师为中心的教学模式一直在外语教学中占主导地位, 很少顾及到个人语言要求, 学习策略及风格等情况, 教师是课堂的主角, 学生只是课堂教学 的配角, 处于被动接受知识的地位。这种只注重知识传授和积累, 脱离实际运用的填鸭式教 学模式, 必然会导致学生学习主动性的缺乏。因此, 必须改变教师的传统角色, 培养学生的 自主性。要培养策略型的自主学习者, 首先必须丰富其元认知知识, 提高其元认知意识, 尤 其是对学习过程中学习内容、任务、方法及学习性质的元认知。

教师在每学期开课之初, 应向学生讲明整个学期的学习内容和教学安排、每个单元的学习内 容和重难点以及大学英语教学大纲对大学生词汇量的具体要求等, 以便帮助学生制订计划合 同, 包括自我评估、设定短期目标和长期目标以及采用何种方式达到自己的目标 (如: 怎样安 排学习时间, 具体学习什么内容以及任务完成与否的奖惩办法等, 以有效地帮助学生对自己 的学习过程进行规划、管理和监控。通过让学生制订学习计划并督促检查, 教师可以把对学 生学习过程的监控从课堂延伸到课外。

\section{3 灵活运用认知策略}

策略运用是指学生在学习外语过程中对其认为有效的学习方法的选择和使用。认知策略是学 习者赖以获得知识和发展能力所运用的各种具体实施途径的微观行为, 它在语言习得中起着 非常重要的作用。调查显示, 传统的学习方法依然起着不可忽视的作用, 而注重运用的学习 理念虽然已经达到一定的效果, 但还有较大的上升空间。正如 O'Malley 所说, 学习者经常依 赖于那些不需要积极脑力活动的学习策略。因此, 在大学英语教学过程中, 我们应注意训练 学习者使用多种认知策略。

3. 3.1 鼓励学生用英语进行交际

在课堂教学过程中, 教师要为学生创造情景, 让学生根据课文内容模仿、扮演各种角色。学 生在真实的教学环境中与他人积极交流, 在实践中反思自己的学习体验, 通过群组中成员间 的积极互动以及他们与老师之间的互动交流, 完成教学目标, 提高学习效果, 从而增强学生 的交际能力。在教学过程中, 教师对学生的语言错误一一特别是语法错误, 采取比较宽容的 态度。因为交际法教学强调意思的相互传递, 语言的自由选择和交际目的的实现。学生说外 语犯了错误, 不一定是坏事, 说明他们正在尝试使用语言。应用语言学界的权威科德认为: 错误对语言学习是重要的。在交际过程中, 如果教师有错必纠会打断学生的思维, 破坏其语 言的流畅性, 更为严重的是可能会使学生因害怕犯错误而不敢大胆地表达自己的意思, 妨碍 学生的自由交际。

\section{3.2 注重文化背景知识的介绍}

背景知识主要是指与语言学习有关的英美等国家语言习惯、风土人情等社会文化背景知识。 不同的国家由于民族、社会制度、地域、职业、性别、年龄等方面的不同而形成语言与文化 的差异都有其各自的特点。由于语言和文化的不同, 在跨文化交际中, 即使语句准确无误, 也会因使用不当而产生误解, 从而达不到交际的目的。同一个词或同一种表达方式, 对于不 同的背景知识的人有不同的意义, 因此不了解背景知识就会影响对语言的理解。

许多学生对以英语为母语国家的文化背景知识缺乏了解, 因而即使看懂了字面意思, 也不能 理解其真正的含义。因此教师在课堂上要详细解释相关的文化背景, 尤其着重讲解由于文化 差异而引起的词汇、语言运用方面的差异。教师可以利用先进的多媒体教学设备, 采用影视 欣赏的办法, 再现真实生活场景, 使学生在愉悦的环境中领悟文化内涵。

3.3.3 提高学生运用语境学习的能力

简而言之, 语境就是语言所处的环境。调查表明: 非英语专业本科生在语言习得时较少运用 语境, 对词汇的深层理解运用不够。因此, 课堂教学要避免采用仅在单词和句子层次上分析 
语法结构的方法去理解词汇的意义, 而训练学生从较大的语言单位 (句群、段落、章节) 和相 对整体语义入手, 根据上下文意思或语境以及词语搭配, 进行判断和联想, 然后得出合乎逻 辑的猜想和推论, 以迅速提高学生对英语的概括理解能力。此外, 应引导学生利用课余时间 广泛阅读; 同时, 还应通过规定任务的方式, 指导学生有选择有目的地阅读。教师还应指导 学生如何利用语境学习词汇, 通过语境推测词义, 从而使学生扩大词汇量, 提高阅读水平。 总之, 教师必须意识到 “授人以鱼莫如授之以渔” 的重要性, 重视对农业院校学生传授学习 策略和技巧。选择一套根据这一思路编写的教材, 利用好有限的课堂时间, 根据教材所提供 的语境和作业练习将学习策略和技巧的传授穿插于语言教学中。教师也可根据学生的薄弱环 节 (如单词记忆、听力理解和阅读速度等方面) 自选材料进行专门训练, 教师还应指导学生对 适合个人的学习策略进行选择, 鼓励学生摸索出行之有效的学习策略与技巧, 实行自主学习。

\section{致谢}

基金项目：第三批中国外语基金项目（029）

\section{References}

[1]. Maclntyre, P. Toward a social psychological mode of strategy use. Foreign language annuals. Vol.27(1994), p.185-195.

[2]. Lightbown, M., Spada, N. How Language Aye Learned. Shanghai. Shanghai foreign language education press, 2002, p.3-18.

[3]. VanPatten, B. From Input to Output: A Teacher's Guide to Second Language Acquisition. Beijing: World Publishing Cooperation. 2007, p.12.

[4]. LIU Yong-an. Empirical Studies on the Cultivation of Autonomous Larning Ability in College English Learning. Overseas English. 2004, No.8, p.72-73, 77.

[5]. Beson, P. Teaching and researching autonomy in language learning. Beijing, China: Foreign Language Teaching and Research Press. 2005, p.56-59.

[6]. HU Shu-lan, LI Hong-ying, WEI Wei. The Study of English Learning Strategies of College Students in Western Area-Based on some Colleges of Gansu Province. Journal of Lanzhou Jiaotong University. Vol.33(2014), No.4, p.150-152.

[7]. Oxford, R. Language Learning Strategies: What Every Teacher Should Know. Rowley, Mass: Newbury House, 1990, p.47-58.

[8]. LI Meng-shu. The Reform and Practice of College English Learning Strategy Instruction. JOURNAL OF SOCIAL SCIENCE OF JIAMUSI UNIVERSITY. Vo1.32(2014), No.2, p.171-173. 